\title{
High Throughput Screening Platform for a FAD-Dependent L-Sorbose Dehydrogenase
}

\begin{abstract}
Xiaoyu Shan ${ }^{1,2,3}$, Li Liu ${ }^{1,2,4}$, Weizhu Zeng ${ }^{1}$, Jian Chen ${ }^{1,2,4}$ and Jingwen Zhou ${ }^{1,2,4 *}$
'School of Biotechnology and Key Laboratory of Industrial Biotechnology, Ministry of Education, Jiangnan University, Wuxi, China, ${ }^{2}$ National Engineering Laboratory for Cereal Fermentation Technology, Jiangnan University, Wuxi, China, ${ }^{3}$ The Key Laboratory of Carbohydrate Chemistry and Biotechnology, Ministry of Education, Jiangnan University, Wuxi, China, ${ }^{4}$ Jiangsu Provisional Research Center for Bioactive Product Processing Technology, Jiangnan University, Wuxi, China
\end{abstract}

2-Keto-L-gulonic acid (2-KLG) is the direct precursor for the production of L-ascorbic acid (L-Asc) on industrial scale. Currently, the production of L-Asc in the industry is a two-step fermentation process. Owing to many unstable factors in the fermentation process, the conversion rate of $\mathrm{L}$-sorbose to $2-K L G$ has remained at about $90 \%$ for many years. In order to further improve the production efficiency of 2-KLG, a FAD-dependent sorbose dehydrogenase $(\mathrm{SDH})$ has been obtained in our previous research. The SDH can directly convert L-sorbose to $2-K L G$ at a very high efficiency. However, the enzyme activity of the $\mathrm{SDH}$ is relatively low. In order to further improve the enzyme activity of the $\mathrm{SDH}$, a high throughput screening platform the dehydrogenase is essential. By optimizing the promoter, host and sorbosone dehydrogenase (SNDH), knockout of the aldosterone reductases and PTS related genes, a reliable platform for high-throughput screening of more efficient FAD-dependent SDH has been established. By using the high-throughput screening platform, the titer of the 2-KLG has been improved by $14.1 \%$. The method established here could be useful for further enhancing the FAD-dependent $\mathrm{SDH}$, which is important to achieve the efficient one-strain-single-step fermentation production of 2-KLG.

Keywords: 2-Keto-L-gulonic acid, promoter, sorbose dehydrogenase, sorbosone dehydrogenase, aldosterone reductase, L-sorbose-specific-PTS, error-prone PCR

\section{INTRODUCTION}

Vitamin C, also known as L-ascorbic acid (L-Asc), is an essential nutrient for human beings and occupies the largest global market among vitamins. It can affect cells by participating in the elimination of reactive oxygen species (Wenzel et al., 2004), promoting collagen production (May and $\mathrm{Qu}, 2005)$, and helping immune defense (Carr and Maggini, 2017). At present, the main L-Asc production method is the classical two-step fermentation process, which uses Gluconobacter oxydans to convert D-sorbitol to L-sorbose, and uses Ketogulonigenium vulgare and Bacillus megaterium to further convert L-sorbose to 2-keto-L-gulonic acid (2-KLG). The process has two fermentation steps and requires two times of sterilization, resulting in high energy consumption and long production period (Liu et al., 2011a,b). Besides, compared with commonly used singlefermentation fermentation, both regulation and breeding of mixed fermentation are more difficult (Kim et al., 2019). To achieve the one-step-single-strain production of 2-KLG is a long pursued goal for the vitamin C industry. 
In order to achieve the one-step-single-strain production of 2-KLG, previous attempts could be divided into several groups: (1) Overexpression of sorbose dehydrogenase (SDH) and sorbosone dehydrogenase (SNDH) from $K$. vulgare in $G$. oxydans or other bacteria (Gao et al., 2014). Since the SDH from $K$. vulgare could also interact with $\mathrm{D}$-sorbitol to form other byproducts, the yield of $2-\mathrm{KLG}$ on $\mathrm{D}$-sorbitol by this method remains to be lower than 65\%. (2) Overexpression of 2,5-diketoD-gluconate reductase from Corynebacterium glutamicum in Erwinia sp. that can product 2,5-diketo-D-gluconate from Dglucose (Anderson et al., 1985). For potential low enzyme activity or cofactor balance issues, 2-KLG titer could not be significantly improved by using the method. (3) Overexpression of SDH and SNDH from G. oxydans T100 in G. oxydans strains that can produce 2-KLG from D-sorbitol (Saito et al., 1997). When expressing the SDH gene from G. oxydans T100 in E. coli, the $\mathrm{SDH}$ cannot catalyze L- sorbose to $2-\mathrm{KLG}$, suggesting that it may be a major limiting step to increase the enzyme activity of this SDH. According to these previous reports, the main problems in achieving one-step-single-strain fermentation of 2KLG include cofactor regeneration (Wang et al., 2016; Kim et al., 2019), identification of key enzymes, competition of intermediate metabolic byproducts (Richter et al., 2009).

In our recent report, we obtained a $G$. oxydans strain by high-throughput screening aided with 2-KLG dehydrogenase (Chen et al., 2019). A FAD-dependent SDH that can convert Lsorbose to 2 -KLG with almost $100 \%$ conversion efficiency has been identified from the strain. However, the enzyme activity of the SDH is very low and can only produce several grams of 2-KLG by different optimization method. Based the previous reports about other SDHs that can convert L-sorbose to Lsorbosone or 2-KLG (Zhou et al., 2012; Gao et al., 2017), both substrate/product specificity and enzyme activity should be improved by high-throughput screening. Since the slow growth rate of G. oxydans and low transformation efficiency (Yao et al., 2017; Jin et al., 2019), G. oxydans itself is not suitable for highthroughput screening of efficient SDH. Therefore, it is essential to construct a high-throughput screening platform strain with fast growth and high transformation efficiency. Since the SDH could be functional expressed in E. coli to catalyze L-sorbose to 2-KLG, E. coli could be a good choice (Chen et al., 2019). Though D-sorbitol, L-sorbose, L-sorbosone and 2-KLG are not commonly acquired in E. coli, the aldosterone reductases and PTS-related proteins could affect the regular function of SDH and thus interrupt the high-throughput screening process.

This study was focused on establishment of a high-throughput screening platform of a FAD-dependent SDH from G. oxydans WSH-003 based on E. coli, and then improve the enzyme activity of the SDH. By optimizing the promoter of the SDH, it was found that introduction of $\mathrm{SDH}$ in $E$. coli not only produce 2-KLG, there are also some byproducts could be found. In order to achieve more reliable screening process, the aldosterone reductases and PTS related genes are knockout consequently, combined with the co-expression of a SNDH. By using the high-throughput screening system, the titer of the 2-KLG has been improved by $14.1 \%$. The method established here could be useful for further enhancing the FAD-dependent SDH,
TABLE 1 | Primers used for promoter optimization.

\begin{tabular}{ll}
\hline Primer & Sequence $\mathbf{5}^{\prime} \mathbf{- 3}^{\prime}$ \\
\hline infC-rplT-F & gcgcggatcttccagagattctgaatacgttaacgaattgacgc \\
infC-rpIT-R & agttcttctcccttacccataccttattcctccaattgtttaagac \\
Ipp-F & gcgcggatcttccagagatttgaatccgatggaagcatcctg \\
Ipp-R & agttcttctcccttacccattattaataccctctagattgagttaatctc \\
cspA-F & cgcggatcttccagagattattgctgtttacggtcctgatg \\
cspA-R & agttcttctcccttacccatagtgtattacctttaataattaagtgtgcc \\
dnaKJ-F & gcggatcttccagagatttcttgtcctgccatatcgcg \\
dnaKJ-R & agttcttctcccttacccatctaaacgtctccactatatattcgg \\
csrA-F & gcgcggatcttccagagatttacctgcagcgttagccagtg \\
csrA-R & agttcttctcccttacccatagtgtattacctttaataattaagtgtgcc \\
SDH-R & gttcttctcccttacccattcaggcgttcccctgaatgaaatc \\
infC-rplT-SDH-F & aacaattggaggaataaggtatgacgagcggtttgattacatcg \\
Ipp-SDH-F & caatctagagggtattaataatgacgagcggttttgattacatcg \\
cspA-SDH-F & attattaaaggtaatacactatgacgagcggttttgattacatcg \\
dnaKJ-SDH-F & tatatagtggagacgtttagatgacgagcggttttgattacatcg \\
csrA-SDH-F & aatctttcaaggagcaaagaatgacgagcggttttgattacatcg \\
\hline
\end{tabular}

which is important to achieve the efficient one-strain-single-step fermentation production of $2-\mathrm{KLG}$.

\section{MATERIALS AND METHODS \\ Genes, Plasmids, and Strains}

E. coli JM109 was used for plasmid construction and preservation. E. coli BL21 (DE3) was used for gene expression research. The plasmids pCas and pTarget required for gene knockout were provided by Shanghai Institute of Plant Physiology and Ecology (Jiang et al., 2015). G. oxydans WSH-004 was screened in our previous research (Chen et al., 2019).

\section{Plasmid Construction and Gene Expression}

Five constitutive promoters with different strength $\left(\mathrm{P}_{\text {infC-rplT }}\right.$, $\mathrm{P}_{\text {lpp }}, \mathrm{P}_{\text {dnaKJ }}, \mathrm{P}_{\mathrm{cspA}}, \mathrm{P}_{\mathrm{csrA}}$ ) have been selected to express $\mathrm{SDH}$ (Zhou et al., 2017). These promoters were obtained by PCR amplification using the E. coli K12 genome as the template. $\mathrm{SDH}$ gene was PCR-amplified from the genomic DNA of $G$. oxydans WSH-004. The target gene $\mathrm{SDH}$ and these promoters were ligated into the vector pMD19-T-Simple by a one-step cloning kit (Takara, Dalian, China). All the primers used for expression of $\mathrm{SDH}$ are listed Table 1. These plasmids have also been transformed into E. coli K12substr. W3110, E. coli K12substr. MG1655, E. coli JM109 for selecting optimum E. coli host.

\section{Culture Conditions}

E. coli strains with plasmids are plated on LB plates with 100 $\mathrm{mg} / \mathrm{L}$ of ampicillin and cultured overnight. A single colony is picked into a $14 \mathrm{~mL}$ shaker tube containing $4 \mathrm{ml} \mathrm{LB}$ medium and then cultured for $9-10 \mathrm{~h}$ as seed culture. The seed culture was then inoculated with an inoculation ratio of $2 \%$ into a $250 \mathrm{~mL}$ shake flask containing $25 \mathrm{~mL}$ of LB medium with 1\% L-sorbose, 
firstly incubated at $37^{\circ} \mathrm{C}$ for $3 \mathrm{~h}$, and then transferred to 25,30 , 37 , and $40^{\circ} \mathrm{C}$, respectively, for optimizing the temperature for enzyme expression.

\section{Simultaneous Overexpression of SDH and SNDH}

For simultaneous overexpression of SDH and SNDH, the highest yielding constitutive promoter $\mathrm{P}_{\text {cspa }}$ was selected as pMD19cspA-SDH. E. coli BL21(DE3) competent cell was simultaneously transfected into pMD19-cspA-SDH and pET28a-SNDH. Both SDH and SNDH were from G. oxydans WSH-004. Single colonies were picked into seed culture medium for $9 \mathrm{~h}$, then transferred to a $250 \mathrm{~mL}$ shake flask containing $25 \mathrm{~mL}$ of LB medium with $1 \%$ L-sorbose by $2 \%$ inoculum, then incubated at $37^{\circ} \mathrm{C}$ for $2-3 \mathrm{~h}$ until about $\mathrm{OD}_{600}=0.6-1$, adding IPTG with a final concentration of $0.3 \mathrm{mM}$, transferring to a $30^{\circ} \mathrm{C}$ shaker for subsequent fermentation, taking samples at different times and used HPLC to detect for 2-KLG yield.

For optimizing sorbosone dehydrogenase (SNDH) from different sources, five SNDH from other sources were synthesize the five SNDH genes. To construct plasmids for overexpression of SNDH in E. coli BL21 (DE3), SNDH genes were inserted into the HindIII/EcoRI site of pET28(a)+. These plasmids containing SNDH werer transformed into E. coli BL21 (DE3) strain containing SDH plasmid to achieve the co-expression of $\mathrm{SDH}$ and SNDH in E. coli.

\section{Knockout of Aldosterone Reductase Genes}

The aldosterone reductase genes, yiaK, ahr, $d \mathrm{kgA}, d \mathrm{kgB}, y a h K$, $y a j O, y d j G$, yeaE, have been amplified from genomic DNA of E. coli BL21 (DE3) by using yiaK-UP-F/R, yiaK-Down-F/R, $a h r$-UP-F/R, $a h r$-Down-F/R, $d k g A$-UP-F/R, $d k g A$-Down-F/R, $d k g B-U P-F / R, \quad d k g B$-Down-F/R, yahK-UP-F/R, yahK-DownF/R, yajO-UP-F/R, yajO-Down-F/R, $y d j G$-UP-F/R, $y d j G$-Down$\mathrm{F} / \mathrm{R}$, yeaE-UP-F/R, yeaE-Down-F/R, respectively, to obtain upstream and downstream homology arm fragments. These genes of homologous arm were obtained by overlapping PCR using PrimerSTAR DNA polymerase. All the primers are listed in Table 2. The pCas plasmid can generate sgRNA located on the pTarget plasmid under IPTG induction, and the pCas plasmid itself will be incubated at $42^{\circ} \mathrm{C}$. A series of pTarget plasmids were constructed containing the target gene N20-sgRNA of aldosterone reductase genes by primer sequences listed in Table 2. Knockout of these genes by CRISPR/Cas9 are performed according to the previous report (Jiang et al., 2015).

\section{Knockout of Related Genes About L-Sorbose-Specific-PTS}

By using the CRISPR/Cas9 technology, continued to knock out six related genes ( $p t s G, f r u A, p f k A, p t s H, p t s I, g l c A$ ) in Lsorbose-specific PTS was based on the original strain E. coli BL21 (DE3) and E. coli BL21 (DE3)-8. A series of pTarget plasmids were constructed containing the target gene N20-sgRNA of aldosterone reductase genes by primer sequences listed in Table 3. Knockout of these genes by CRISPR/Cas9 are performed according to the previous report (Jiang et al., 2015). Using the
TABLE 2 | Primers used for knockout aldosterone reductases.

\begin{tabular}{|c|c|}
\hline Primer & Sequence $5^{\prime}-3^{\prime}$ \\
\hline yahK-UP-F & ccagggagtggggcaatctgaatatg \\
\hline yahK-UP-R & attatgtggcgcagctactgtattccgccccacaatttcatgacccggca \\
\hline yahK-Down-F & tgccgggtcatgaaattgtggggcggaatacagtagctgcgccacataat \\
\hline yahK-Down-R & ccaggcactatcagaaatcgctcat \\
\hline yahK-sgRNA-F & gtggctcctttgttgtgtgcgttttagagctagaaatagcaagtt \\
\hline yahK-sgRNA-R & gcacacaacaaaggagccacactagtattatacctaggactgagc \\
\hline dkgA-UP-F & aggaggaacgtatggctaatccaac \\
\hline dkgA-UP-R & cttccggctctgcatgatgatgtccggtgatggatctggaagttgcacac \\
\hline$d k g A-D o w n-F$ & gtgtgcaacttccagatccatcaccggacatcatcatgcagagccggaag \\
\hline dkgA-Down-R & gctgccatgattgctgacaatatc \\
\hline dkgA-sgRNA-F & ccattagcgcaaggagggaagttttagagctagaaatagcaagtt \\
\hline dkgA-sgRNA-R & ttccctccttgcgctaatggactagtattatacctaggactgagc \\
\hline$d k g B-U P-F$ & gccagaatcgcaaaaatcctctgca \\
\hline dkgB-UP-R & agaggcttaatcccattcaggagcccggccagtggattagagtcagatca \\
\hline dkgB-Down-F & tgatctgactctaatccactggccgggctcctgaatgggattaagcctct \\
\hline dkgB-Down-R & gcgctggtacgttaacggattcca \\
\hline$d k g B$-sgRNA-F & gaagggttgacgcgtgagatgttttagagctagaaatagcaagtt \\
\hline dkgB-sgRNA-R & atctcacgcgtcaacccttcactagtattatacctaggactgagc \\
\hline ahr-UP-F & ggctggaacgcttaaatgatgcttc \\
\hline ahr-UP-R & gcggtaatcagatcaactgcgagcacttatgagctgcgtaagctgatgcg \\
\hline ahr-Down-F & cgcatcagcttacgcagctcataagtgctcgcagttgatctgattaccgc \\
\hline ahr-Down-R & cgttgtggattatacctgtcgcacg \\
\hline ahr-sgRNA-F & caaaatagggctgccagtcggttttagagctagaaatagcaagtt \\
\hline ahr-sgRNA-R & cgactggcagccctattttgactagtattatacctaggactgagc \\
\hline yeaE-UP-F & cgcgtgtatctgaatccacaaga \\
\hline yeaE-UP-R & ggcattgaactcggtttaaccctcacaatatcagcgcggcacaagtattg \\
\hline yeaE-Down-F & caatacttgtgccgcgctgatattgtgagggttaaaccgagttcaatgcc \\
\hline yeaE-Down-R & cgggtattggtgtgcagtggaac \\
\hline yeaE-sgRNA-F & atctgctgttgctggcaccagttttagagctagaaatagcaagtt \\
\hline yeaE-sgRNA-R & tggtgccagcaacagcagatactagtattatacctaggactgagc \\
\hline уајО-UP-F & tcggcgtctatctttgtcatcagac \\
\hline уајO-UP-R & gtaatcacgcatggacactgccggaattatcggtacatcgcgggaagaac \\
\hline yajO-Down-F & gttcttcccgcgatgtaccgataattccggcagtgtccatgcgtgattac \\
\hline yajo-Down-R & tctggaaatggccaccagccatg \\
\hline yajO-sgRNA-F & gcccggtttactcaacaaccgttttagagctagaaatagcaagtt \\
\hline yajO-sgRNA-R & ggttgttgagtaaaccgggcactagtattatacctaggactgagc \\
\hline ydjG-UP-F & tcatcttccagcttctcaagatcgc \\
\hline ydjG-UP-R & cctttaggcacaacggatattacgc ccgctatgtcgtgataatggcattg \\
\hline ydjG-Down-F & caatgccattatcacgacatagcgg gcgtaatatccgttgtgcctaaagg \\
\hline ydjG-Down-R & acaaaccgtaacggcagtctgtggg \\
\hline ydjG-sgRNA-F & agctggcttctacctcttcggttttagagctagaaatagcaagtt \\
\hline ydjG-sgRNA-R & cgaagaggtagaagccagctactagtattatacctaggactgagc \\
\hline yiak-UP-F & ccatgtagatcttgcccattgcg \\
\hline yiak-UP-R & gcgcgatatgtccaaaaatcatgacctcaaatgtcactttcatcccaggc \\
\hline yiaK-Down-F & gcctgggatgaaagtgacatttgaggtcatgattttggacatatcgcgc \\
\hline yiak-Down-R & ttcaagtcgcatgtgcagcaacct \\
\hline yiaK-sgRNA-F & ggcgcaaaagagtgtcgcatgttttagagctagaaatagcaagtt \\
\hline yiaK-sgRNA-R & atgcgacactcttttgcgccactagtattatacctaggactgagc \\
\hline
\end{tabular}

E. coli BL21 (DE3) genome as a template, respectively, amplify using PrimerSTAR DNA polymerase, using $p t s G-\mathrm{UP}-\mathrm{F} / \mathrm{R}$, ptsG-Down-F/R, fruA-UP-F/R, fruA-Down-F/R, glcA-UP-F/R, 
glcA-Down-F/R, ptsI-ptsH-UP-F/R, ptsI-ptsH-Down-F/R, as a pair primer to obtain upstream and downstream homology arm fragments. These genes of homologous arm were obtained by overlapping PCR using PrimerSTAR DNA polymerase. All the primers are listed in Table 3.

\section{Error-Prone PCR}

Error-prone PCR of SDH was performed by the GeneMorph II Random Mutagenesis Kit (Agilent, Santa Clara, CA) with pMD19-cspA-SDH as a template by using primer pair $5^{\prime}$-TTC CAGAGATTATTGCTGTTTACGG-3' $/ 5^{\prime}$-GTGAAAAGTTCT

TCTCCCTTACCCA-3'. The amplified fragment carrying A tail was directly cloned with the vector pMD19-Simple transformed into E. coli BL21 (DE3) derivative strains mentioned above. For primary screening, the colonies appeared on the LB plates with ampicillin were picked up into 96-deep well plates by QPix420 (MD, Genetix, UK). These cultured cells in the deep-well plate were cultured at $37^{\circ} \mathrm{C}$ for $2-3 \mathrm{~h}$, then moved to culture at $30^{\circ} \mathrm{C}$ for $20-24 \mathrm{~h}$. For high-throughput screening, $40 \mathrm{uL}$ of supernatant was transferred into another 96-well plate, then

TABLE 3 | Primers used for knockout of PTS related genes.

\begin{tabular}{|c|c|}
\hline Primer & Sequence $5^{\prime}-3^{\prime}$ \\
\hline ptsG-UP-F & actcaccttaccttgcgccggtacc \\
\hline ptsG-UP-R & gtaccgaaaatcgcctgaacaccagaaccgcctgcttctgccataacatg \\
\hline ptsG-Down-F & catgttatggcagaagcaggcggttctggtgttcaggcgattttcggtac \\
\hline ptsG-Down-R & ggcgcaattaccgacaactggcagc \\
\hline$p t s G-s g R N A-F$ & tccttcatttggccgccgatgttttagagctagaaatagcaagtt \\
\hline ptsG-sgRNA-R & atcggcggccaaatgaaggaactagtattatacctaggactgagc \\
\hline fruA-UP-F & caacgccaggttttgtgcaatatt \\
\hline fruA-UP-R & cggtaaaaatgtctggctgggtgatagcgaaagcagcgtaataaaaggtg \\
\hline fruA-Down-F & caccttttattacgctgctttcgctatcacccagccagacatttttaccg \\
\hline fruA-Down-R & tgaaacctaaccgccgcgagctgga \\
\hline fruA-sgRNA-F & aaactgatggcaccacacgggttttagagctagaaatagcaagtt \\
\hline fruA-sgRNA-R & ccgtgtggtgccatcagtttactagtattatacctaggactgagc \\
\hline$g / c A-U P-F$ & cggcatcactgatagaaaaacaggtg \\
\hline$g / c A-U P-R$ & gacatgtcgctggagcaataaccctatcgaccacggcgcagcaaatcaac \\
\hline glcA-Down-F & gttgatttgctgcgccgtggtcgatagggttattgctccagcgacatgtc \\
\hline glcA-Down-R & gcgaaagtggttgatcagcaaaacg \\
\hline$g / c A-s g R N A-F$ & agaacggcacaagaaccgacgttttagagctagaaatagcaagtt \\
\hline$g / c A-s g R N A-R$ & gtcggttcttgtgccgttctactagtattatacctaggactgagc \\
\hline pfkA-UP-F & ttgatatcgtgacttcctggccggg \\
\hline pfkA-UP-R & gctgttcgttctggatacctacgcacgctgtaacggtctagctgtaccat \\
\hline pfkA-Down-F & atggtacagctagaccgttacagcgtgcgtaggtatccagaacgaacagc \\
\hline pfkA-Down-R & actgttcgtacaattcgcgcgttgg \\
\hline pfkA-sgRNA-F & gaagtgatgggccgttattggttttagagctagaaatagcaagtt \\
\hline$p f k A-s g R N A-R$ & caataacggcccatcacttcactagtattatacctaggactgagc \\
\hline ptsl-ptsH-UP-F & gcaggtatctcttctggagcagctg \\
\hline ptsl-ptsH-UP-R & ccagcgtcattaactcgtccgttgtagcggtaatggtaacttcttgctgg \\
\hline ptsl-ptsH-Down-F & ccagcaagaagttaccattaccgctacaacggacgagttaatgacgctgg \\
\hline ptsl-ptsH-Down-R & gcgaaagtggttgatcagcaaaacg \\
\hline ptsl-ptsH-sgRNA-F & gttgtgactatctccgcagagttttagagctagaaatagcaagtt \\
\hline ptsl-ptsH-sgRNA-R & tctgcggagatagtcacaacactagtattatacctaggactgagc \\
\hline
\end{tabular}

mixed with buffer containing $2-\mathrm{KLG}$ reductase and NADH to a total volume $200 \mathrm{uL}$. The last one of each 96-well plate was set as a control. The absorbance at $340 \mathrm{~nm}$, which is the optimum absorbance for $\mathrm{NADH}$, was detected by a microplate reader (BioTek, Winooski, VT).

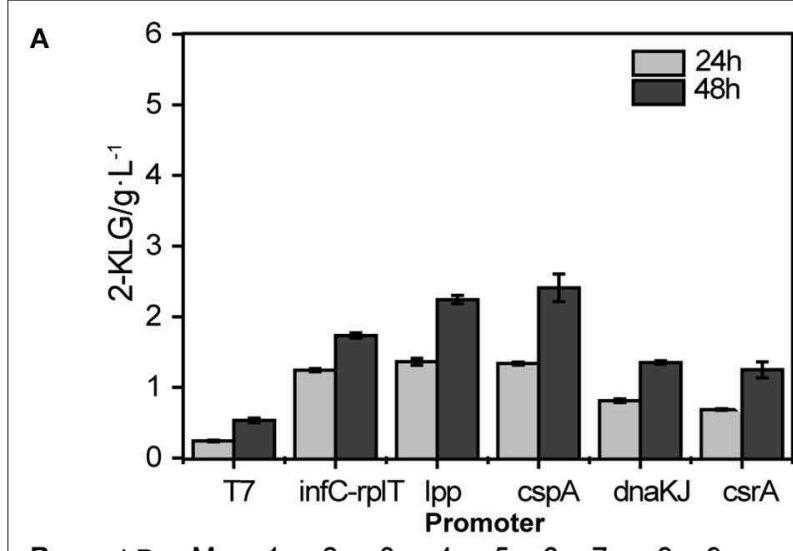

B
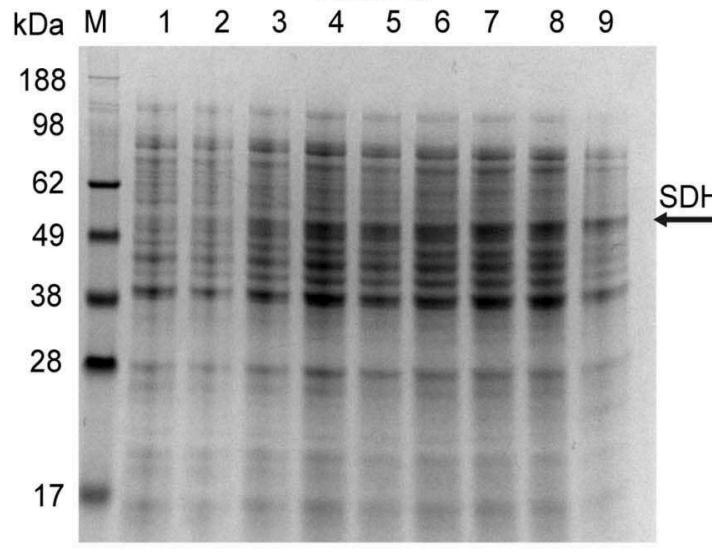

C

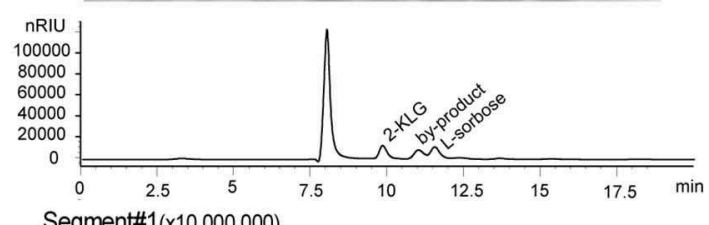

D
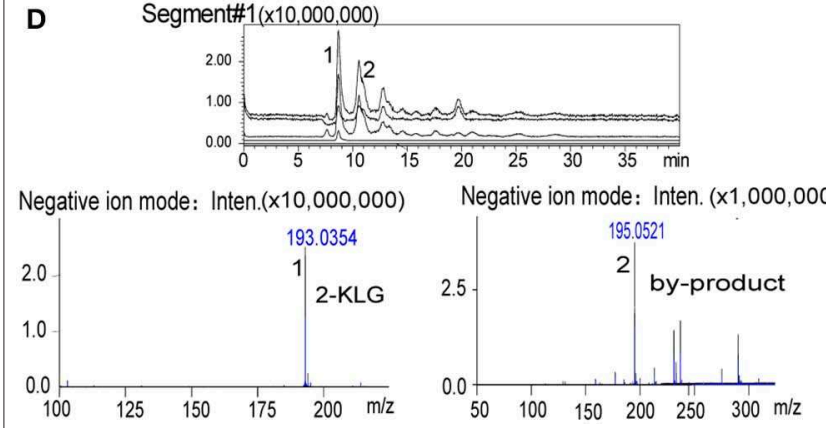

FIGURE 1 | Optimized different constitutive promoter for the expression of SDH. (A) Effect of different promoter on 2-KLG production by HPLC analysis. (B) Whole cell SDS-PAGE analysis M: protein Marker, 1: blank control, and 2-9 represent constitutive expression of SDH 4, 6, 8, 10, 12, 16, 20, and 24h, respectively. (C) HPLC detection of product peaks (D) LC-MS analysis of product peaks. 
HPLC and Liquid Chromatography Ion Trap Time-Of-Flight Mass Spectrometry

\section{(LCMS-IT-TOF) Assays}

L-sorbose and 2-KLG were determined by a HPLC equipped with an Aminex HPX-87H column (Bio-Rad, Hercules, $\mathrm{CA}$ ) at $35^{\circ} \mathrm{C}$ with a flow rate of $0.5 \mathrm{~mL} / \mathrm{min}$ and $5 \mathrm{mmol} / \mathrm{L} \mathrm{H}_{2} \mathrm{SO}_{4}$ as the eluent (Gao et al., 2014). For LC-MS analysis, a Shimadzu LCMS-ITTOF (Shimadzu, Kyoto, Japan) equipped with an Aminex HPX$87 \mathrm{H}$ column was used. The HPLC conditions for LCMS-IT-TOF analysis is: $35^{\circ} \mathrm{C}$ with a flow rate of $0.5 \mathrm{~mL} / \mathrm{min}$ and $5 \mathrm{mmol} / \mathrm{L}$ formic acid as the eluent. IT-TOF detection was performed with an ESI source in negative ion mode at the followed conditions: detector voltage, $1.60 \mathrm{kV}$; nebulizing gas (N2) flow, $1.5 \mathrm{~L} / \mathrm{min}$; drying gas (N2) flow, $200 \mathrm{kPa}$; ion accumulation time, $30 \mathrm{~ms}$; and scan range ( $\mathrm{m} / \mathrm{z}), 100-300$ for MS1(Chen et al., 2019).

\section{RESULTS}

\section{Overexpression of the FAD-Dependent L-Sorbose Dehydrogenase in E. coli}

Five E. coli BL21 (DE3) strains that express the FAD-dependent SDH with different promoters were constructed and applied for the shake flask culture to produce 2-KLG with $10 \mathrm{~g} / \mathrm{L}$ of L-sorbose as a substrate. The sampled culture broth was then detected by HPLC (Figure 1A). T7 and five constitutive promoters were applied to optimize the expression of SDH in E. coli. The results showed that the promoter $\mathrm{P}_{\text {cspA }}$ could achieve to $2.42 \mathrm{~g} / \mathrm{L}$, which is the highest 2-KLG titer among the strains. SDS-PAGE results showed that the promoter $\mathrm{P}_{\mathrm{cspA}}$ could significantly express $\mathrm{SDH}$, which has a molecular weight of $\mathrm{SDH}$ was $57.6 \mathrm{kDa}$ (Figure 1B). Since both the titer and the conversion ratio is relatively low, it cannot be directly applied for the high-throughput screening of the SDH. The HPLC and LC-MS results showed that besides the L-sorbose and 2-KLG, there is also an unknown byproduct (Figures 1C,D). This by-product was produced in the middle of the fermentation stage. As the fermentation progresses, the byproduct gradually decreased. At the end of the fermentation, the by-product peak was almost disappeared.

\section{Optimized Production of 2-KLG From L-Sorbose}

By expressing the SDH with the optimum promoter $\mathrm{P}_{\text {cspA }}$ in different $E$. coli strains, it was found that SDH could convert L-sorbose to 2-KLG in E. coli K-12 substr. W3110, E. coli
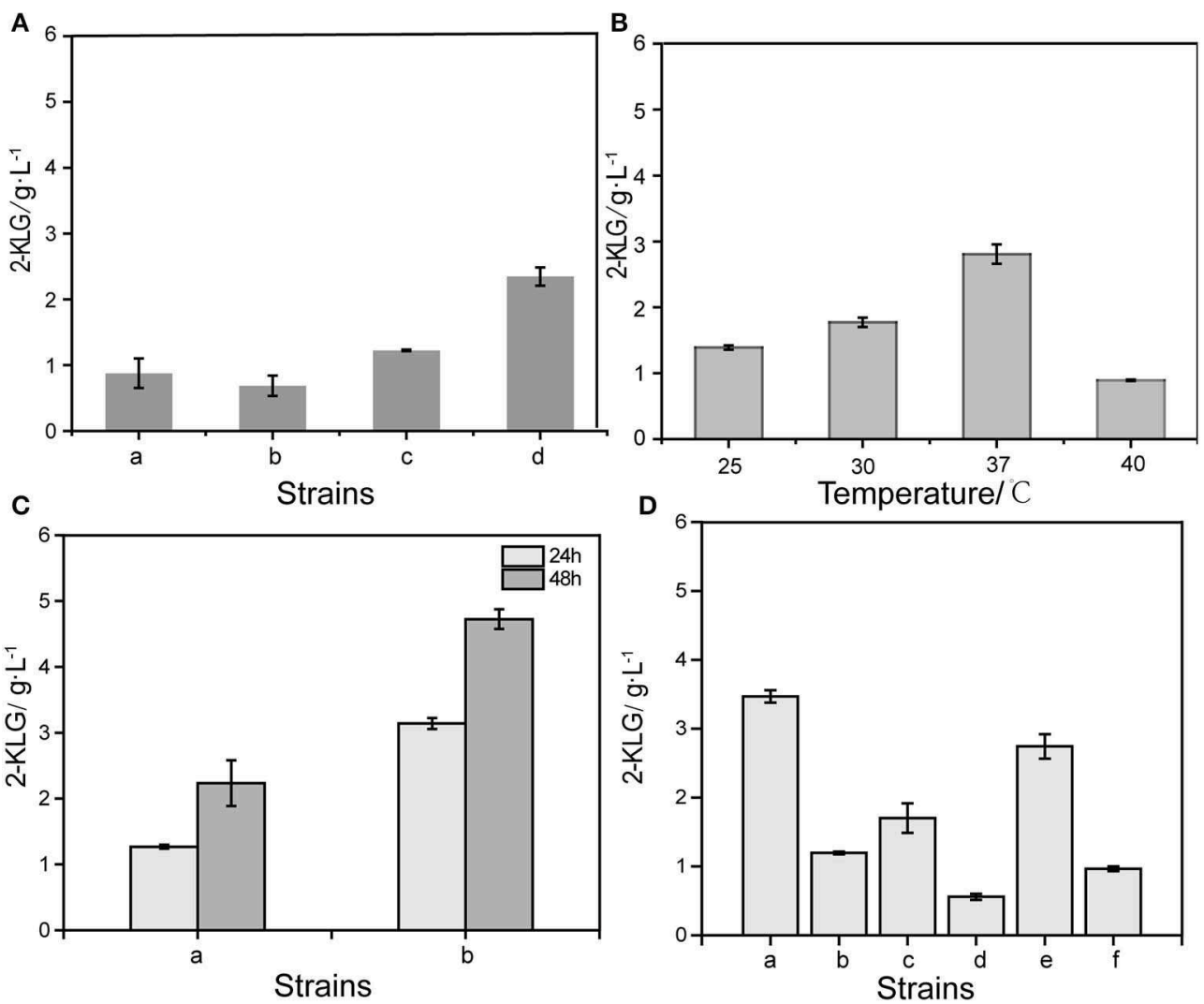

FIGURE 2 | Optimized different conditions of 2-KLG yield from L-sorbose. (A) Optimized host cells for expression of sorbose dehydrogenase. a: E.coli JM109, b: E.coli K-12substr.W3110, c: E.coli K-12substr.MG1655, d: E.coli BL21(DE3). (B) Optimized the effect of culture temperature on the yield of 2-KLG. (C) Effect of co-expression of SDH/SNDH on 2-KLG production. a: Control, b: T-cspA-SDH/pET28a-SNDH. (D) Optimized the effects of different sources of sorbosone dehydrogenase (SNDH) on the yield of 2-KLG. a: sndh-WSH-004, b: sndh-02655, c: sndh-02935, d: sndh-03750, e: sndh-04500, f: sndh-19405. 
TABLE 4 | Strains deficient in aldosterone reductases and PTS system.

\begin{tabular}{|c|c|}
\hline Strains & Genotypes \\
\hline E. coli BL21 (DE3)-3 & $\Delta$ ptsG: $\Delta$ fruA: $\Delta g / c A$ \\
\hline E. coli BL21 (DE3)-4 & $\Delta$ ptsG: $\Delta$ fruA: $\triangle$ glcA: $\Delta p f k A$ \\
\hline E. coli BL21 (DE3)-6 & $\Delta p t s G: \Delta f r u A: \Delta g l c A: \Delta p f k A \Delta p t s H: \Delta p t s l$ \\
\hline E. coli BL21 (DE3)-8 & $\begin{array}{l}\Delta d k g B: \Delta \text { ahr: } \Delta \text { yajO: } \Delta \text { yiaK: } \Delta \text { yahK: } \Delta \text { ydjG: } \Delta \text { yeaE: } \\
\Delta d k g A\end{array}$ \\
\hline $\begin{array}{l}\text { E. coli } \\
\text { BL21(DE3)-8-SDH }\end{array}$ & $\begin{array}{l}\Delta d k g B: \Delta \text { ahr: } \Delta \text { yajO: } \Delta \text { yiaK: } \Delta \text { yahK: } \Delta \text { ydjG: } \Delta \text { yeaE: } \\
\Delta d k g A, \mathrm{SDH}\end{array}$ \\
\hline $\begin{array}{l}\text { E. coli BL21(DE3)-8- } \\
\text { SDH-SNDH }\end{array}$ & $\begin{array}{l}\Delta d k g B: \Delta \text { ahr: } \Delta \text { yajO: } \Delta \text { yiaK: } \Delta \text { yahK: } \Delta \text { ydjG: } \Delta \text { yeaE: } \\
\Delta d k g A, \text { SDH, SNDH }\end{array}$ \\
\hline $\begin{array}{l}\text { E. coli BL21 } \\
\text { (DE3)-11 }\end{array}$ & $\begin{array}{l}\Delta d k g B: \Delta a h r: \Delta y a j O: \Delta y i a K: \Delta y a h K: \Delta y d j G: \Delta y e a E: \\
\Delta d k g A: \Delta p t s G: \Delta \text { fruA: } \Delta g l c A\end{array}$ \\
\hline $\begin{array}{l}\text { E. coli BL21 } \\
\text { (DE3)-12 }\end{array}$ & $\begin{array}{l}\Delta d k g B: \Delta a h r: \Delta y a j O: \Delta y i a K: \Delta y a h K: \Delta y d j G: \Delta y e a E: \\
\Delta d k g A: \Delta p t s G: \Delta \text { fruA: } \triangle g l c A: \Delta \text { pfkA }\end{array}$ \\
\hline $\begin{array}{l}\text { E. coli BL21 } \\
\text { (DE3)-13 }\end{array}$ & $\begin{array}{l}\Delta d k g B: \Delta a h r: \Delta y a j O: \Delta y i a K: \Delta y a h K: \Delta y d j G: \Delta y e a E: \\
\Delta d k g A: \Delta p t s G: \Delta f r u A: \Delta g l c A: \Delta \text { ptsH: } \Delta \text { ptsl }\end{array}$ \\
\hline $\begin{array}{l}\text { E. coli BL21 } \\
\text { (DE3)-14 }\end{array}$ & $\begin{array}{l}\Delta d k g B: \Delta a h r: \Delta y a j O: \Delta y i a K: \Delta y a h K: \Delta y d j G: \Delta y e a E: \\
\Delta d k g A: \Delta p t s G: \Delta \text { fruA: } \Delta g l c A: \Delta \text { pfkA: } \Delta \text { ptsH: } \Delta \text { ptsl }\end{array}$ \\
\hline
\end{tabular}

K-12 substr. MG1655, E. coli JM109 and E. coli BL21 (DE3) (Figure 2A). The result showed that the SDH could yield highest 2-KLG titer in E. coli BL21 (DE3), while the 2-KLG titers in the other three strains are low. Then the E. coli BL21 (DE3) was selected as the host for the downstream experiments. The $E$. coli BL21 (DE3) strain with the plasmid pMD19-cspA-SDH was subjected to shake flask fermentation at 4 different temperatures to obtain the optimum temperature for enzyme expression (Figure 2B). The results showed that the optimum temperature for 2 -KLG production was $37^{\circ} \mathrm{C}$, under which the 2-KLG titer could reached to $2.83 \mathrm{~g} / \mathrm{L}$. Furthermore, when simultaneous expression of pMD19-cspA-SDH and pET28a-SNDH in E. coli BL21 (DE3), the 2-KLG titer could be further improved to 2.54 $\mathrm{g} / \mathrm{L}$ at $48 \mathrm{~h}$ (Figure 2C). Base on the results, different SNDH have been co-expressed. The results showed that co-expression of SNDH from WSH-004 could achieve the highest 2-KLG titer (Figure 2D).

\section{Effects of Aldosterone Reductases Knockout on 2-KLG Production in E. coli}

By knocking out the 8 aldosterone reductase gene using the CRISPR/Cas9 technology, an E. coli strain without aldosterone reductases, E. coli BL21(DE3)-8 (Table 4) has been obtained. Then the SDH or SDH/SNDH was transferred into the aldosterone reductases defective strain to form $E$. coli BL21(DE3)-8-SDH and E. coli BL21(DE3)-8-SDH-SNDH. By culture of $E$. coli BL21(DE3)-8-SDH in shake flasks using 10 $\mathrm{g} / \mathrm{L}$ of L-sorbose as the substrate, the 2-KLG titer was slightly decreased from 2.58 to $2.32 \mathrm{~g} / \mathrm{L}$, while the $2-\mathrm{KLG}$ titer of $E$. coli BL21(DE3)-8-SDH-SNDH was increased from 4.43 to $5.85 \mathrm{~g} / \mathrm{L}$. The results suggested that the aldosterone reductases could play important roles in the function of SDH and SNDH.
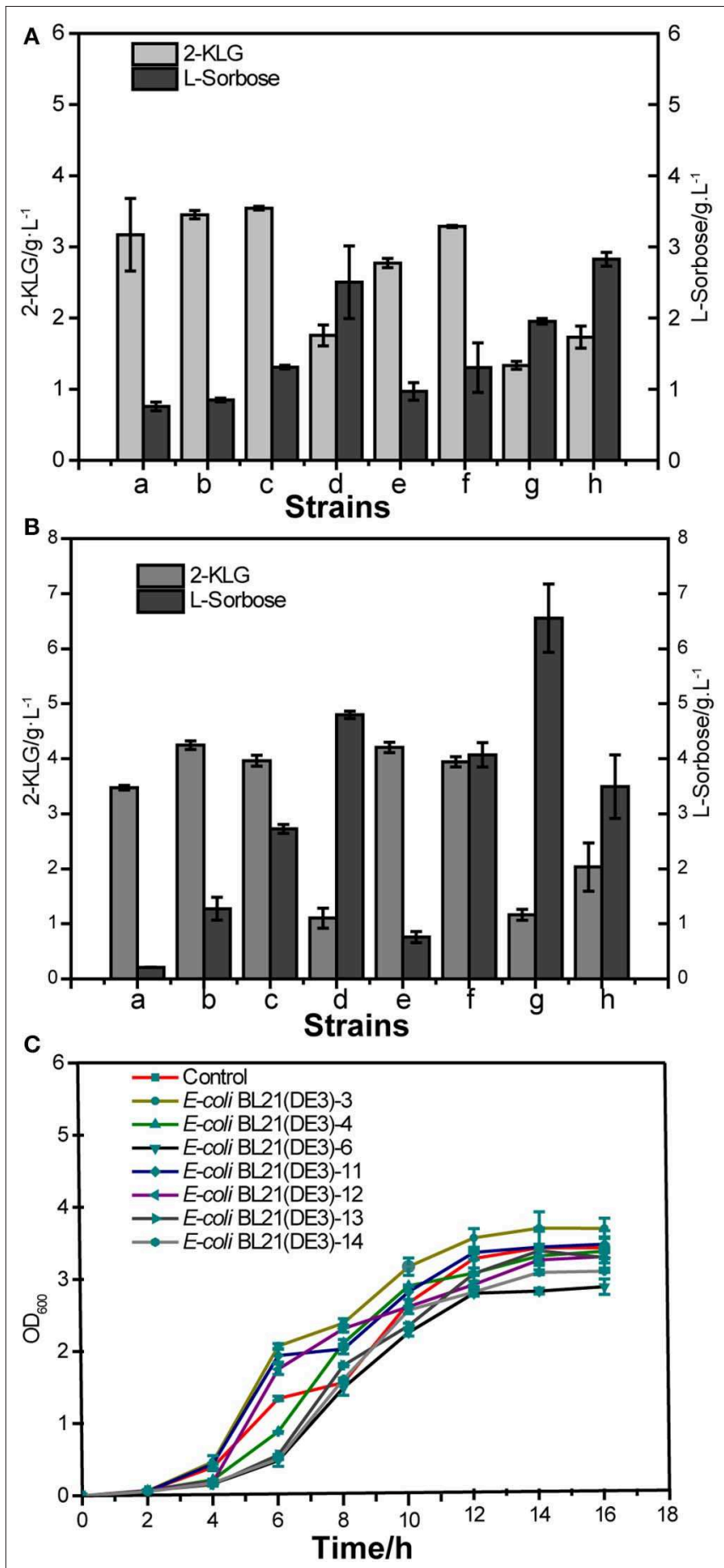

FIGURE 3 | Effects of PTS system proteins knockout on 2-KLG production in E. coli. (A) Overexpression of sorbose dehydrogenase $(\mathrm{SDH})$ in defective strains. a: WT E-coli BL21(DE3), b: E-coli BL21(DE3)-3, c: E-coli BL21(DE3)-4, d: E-coli BL21(DE3)-6; e: E-coli BL21(DE3)-11, f: E-coli BL21(DE3)-12, g: E-coli BL21(DE3)-13, h: E-coli BL21(DE3)-14. (B) Co-expressed sorbose dehydrogenase and sorbosone dehydrogenase (SDH/SNDH) in defective strains. a: Control, b: E-coli BL21(DE3)-3, c: E-coli BL21(DE3)-4, d: E-coli BL21(DE3)-6; e: E-coli BL21(DE3)-11, f: E-coli BL21(DE3)-12, g: E-coli BL21(DE3)-13, h: E-coli BL21(DE3)-14. (C) Defective strain cell growth $\mathrm{OD}_{600}$ detection. 

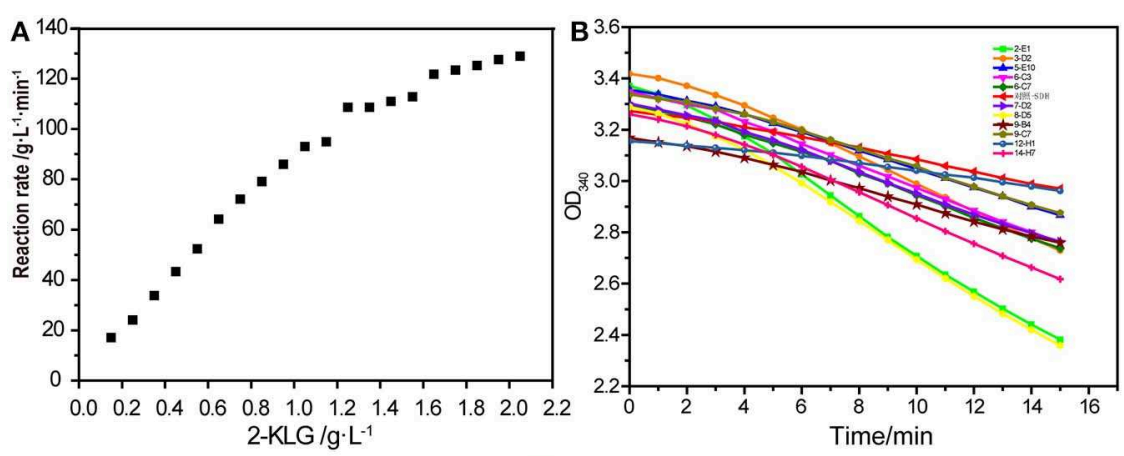

C
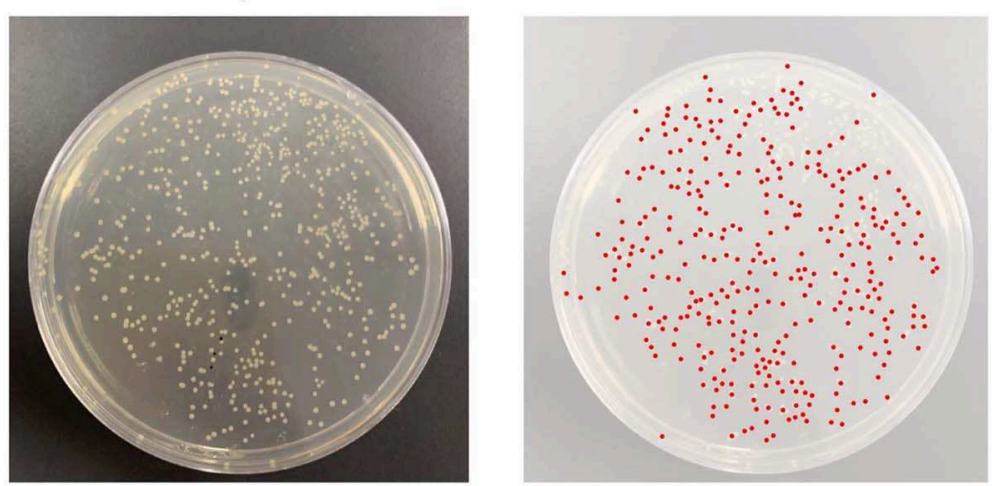

D
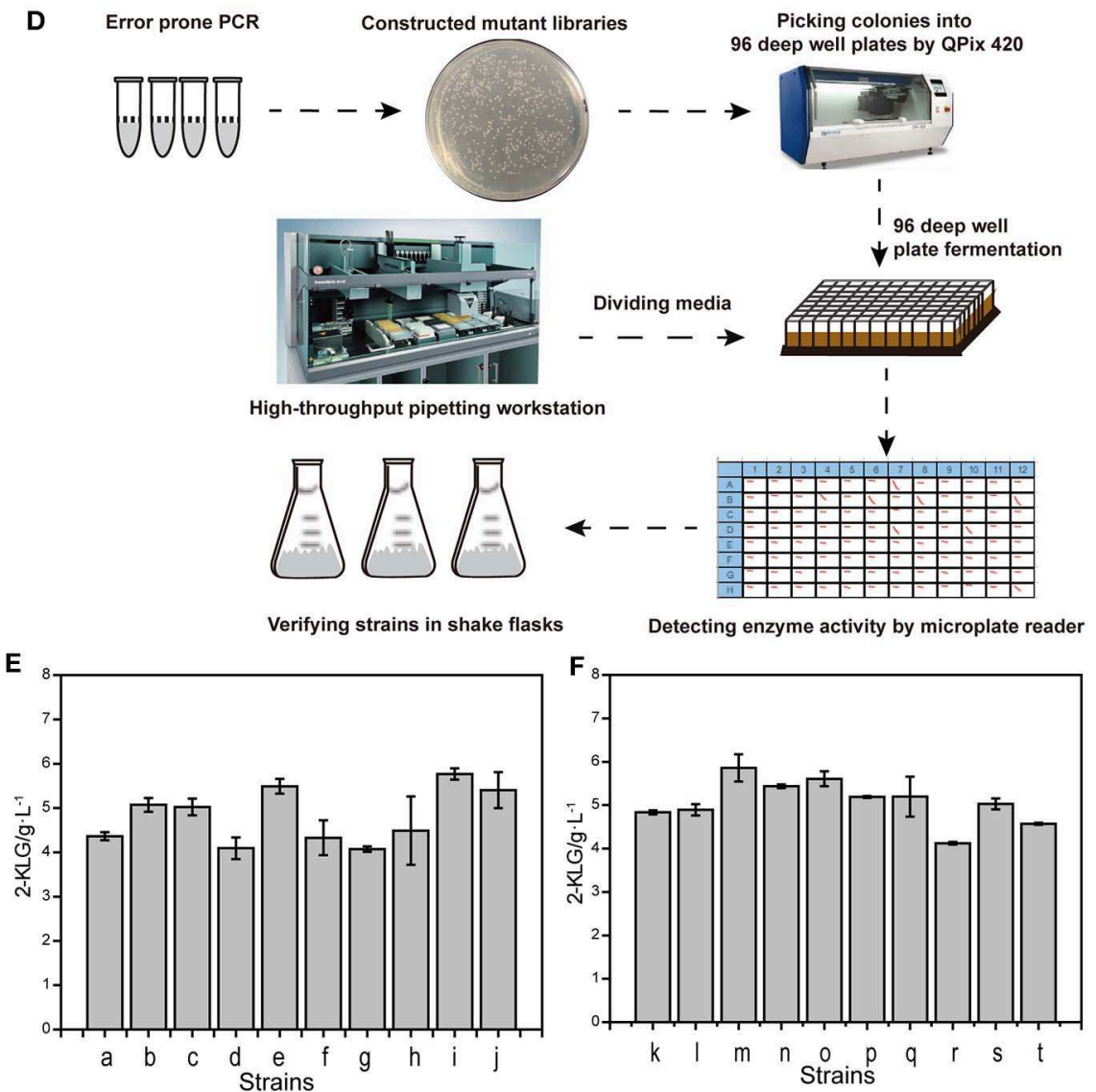

FIGURE 4 | High-throughput screening system. (A) Reaction rate of 2-KLG reductase with different concentrations of 2-KLG. (B) High-throughput preliminary screening visual map based on microplate reader. (C) A typical colony EP-PCR result. (D) The workflow of high-throughput screening method. (E) Verify of the strains in shake flasks (batch 1). a: Control, b: co-2-E1, c: co-8-D5, d: co-9-B4, e: co-6-F7, f: co-6-C3, g: co-5-E10, h: co-3-D2, i: co-15-A7, j: co-16-B6. (F) Verify of the strains in shake flasks (batch 2). k: Control, I: co-20-A1, m: co-28-B1, n: co-19-C1, o: co-15-D1, p: co-33-E1, q: co-6-F1, r: co-8-G1, s: co-25-H1, t: co-5-H5. 


\section{Effects of PTS System Proteins Knockout on 2-KLG Production in E. coli}

Seven PTS system proteins knockout strains were constructed to evaluate the effect of these proteins on the 2-KLG production, i. e., E. coli BL21 (DE3)-3, E. coli BL21 (DE3)-4, E. coli BL21 (DE3)-6, E. coli BL21 (DE3)-11, E. coli BL21 (DE3)12, E. coli BL21 (DE3)-13, E. coli BL21 (DE3)-14 (Table 4). Then SDH or SDH/SNDH were overexpressed in these strains, respectively. The results of shake flask fermentation showed that knockout of $p t s H$ and $p t s I$ could significantly improve the 2KLG titer in strains either overexpression of SDH or SDH/SNDH (Figures 3A,B). The results also showed that knocking out $p t s H$ and $p t s I$ had a great effect on the growth of the cells in the early stage, while the cell growth could achieve the similar level to that of the control strain (Figure 3C).

\section{High Throughput Screening of Sorbose Dehydrogenase in E. coli}

It was determined that the optimal limited range for detection of $2-\mathrm{KLG}$ with $2-\mathrm{KLG}$ reductase was $0-0.23$ (g/L) (Figure 4A). A series of mutants was obtained by using the mutation kit. The initial screening was performed by using microplate reader (Figure 4B). Around $1.3 \times 10^{4}$ mutants were screened for each round. Figure 4C showed one typical colony EP-PCR result by using the workflow shown in Figure 4D. The optimum mutants obtained by preliminary screening were subjected for rescreening with shake flasks. Six strains with higher 2-KLG titer was obtained by rescreening (Figures 4E,F), namely co-2-E1, co16-B6, co-8-D5, co-6-F7, co-15-A7, co-28-B1, increased by 7.1,

TABLE 5 | Mutation sites in SDHs.

\begin{tabular}{ll}
\hline Mutants & Mutation sites \\
\hline co-2-E1 & $\begin{array}{l}\text { Cys17Arg, Gly134Asp, Ile234Val, Val280Met, Trp316Arg, } \\
\text { Thr325Asn } \\
\text { co-16-B6 }\end{array}$ \\
co-8-D5 & Leu44GIn, Glu66Gly, Tyr78Phe, Gly141Val, Asn147lle, \\
& Val159Ala, lle221Phe, Arg273His, Thr304Ala, \\
& Asp407Val, Val490Asp \\
co-6-F7 & Thr443lle, Gly276Asp \\
co-15-A7 & His59Gln, Glu243Gly, Met326Leu, Ser349Pro \\
co-28-B1 & Lys53Arg, Thr65Ala, Thr96Ala, Asp118His, Val119Asp, \\
& Ser146Thr, Asn152Thr, Ile234Asn, Asn236Asp, \\
& Ser348Thr, Gly377Trp, Ser510Thr
\end{tabular}

$10.4,6.6,11.3,14.1$, and $10.2 \%$, respectively. The specific amino acid mutations of these beneficial mutants were shown in Table 5. The results showed that the method established here can be used for high-throughput screening of enhanced SDH. In theory, when the GeneMorph II Random Mutagenesis Kit could yield the similar the probability of mutation of the target gene to A, T, G, and $\mathrm{C}$ base. However, $80 \%$ of the target gene was mutated to $\mathrm{A}$ or $\mathrm{T}$ base in the screened SDH, indicating that the gene may have a preference for A or $\mathrm{T}$ bases.

\section{DISCUSSION}

$\mathrm{SDH}$ with high substrate/product specificity and high enzyme activity is vital for achieving one-step-single-strain production of 2-KLG from D-sorbitol. A FAD-dependent SDH obtained by our previous work has high substrate/product specificity while low enzyme activity was used to improve the enzyme activity by highthroughput screening. By optimizing the promoter, hosts and SNDHs, knockout of the aldosterone reductases and PTS related genes, a reliable platform for high-throughput screening of more efficient FAD-dependent SDH has been established. By using the high-throughput screening system, the titer of the 2-KLG has been improved by $14.1 \%$.

At present, most of the recent researches on the onestep-single-strain production of L-Asc were carried out in the G. oxydans (Table 6) (Wang et al., 2018). The SDH has a similar sequence to that of the G. oxydans T100, which could directly convert D-sorbitol to 2-KLG (Saito et al., 1997). When overexpression of the SDH from G. oxydans T100 in another G. oxydans G624, the titer of 2-KLG could achieve to $130 \mathrm{~g} / \mathrm{L}$ (Saito et al., 1997). However, when synthesize the SDH and SNDH from $G$. oxydans $\mathrm{T} 100$ in $G$. oxydans strains available in our lab, no more than $10 \mathrm{~g} / \mathrm{L}$ of $2-\mathrm{KLG}$ could be obtained. Unlike a majority of the common enzymes, these SDHs from $G$. oxydans are highly hypercritical to strains without any known disciplines according to our experiments, such as: (1) The SDHs from one $G$. oxydans strain cannot be functional in other $G$. oxydans strains and other common bacteria; (2) E. coli strains express the SDHs could have the ability to convert L-sorbose to 2-KLG, while the broken cells cannot, even with common exogenous electron acceptors for dehydrogenases (DCIP, PMS). These strange phenomena significantly affect the further rational engineering of the SDHs from G. oxydans. Since the slow growth rate of G. oxydans and low transformation efficiency (Yao et al.,

TABLE 6 | Production of -KLG by different microorganisms.

\begin{tabular}{|c|c|c|c|c|}
\hline Microorganisms (year) & Substrate & Concentration $(g / L)$ & Titer $(\mathbf{g} / \mathrm{L})$ & $\begin{array}{c}\text { Yield } \\
\text { (mol/ } \mathrm{mol})\end{array}$ \\
\hline G. oxydans T-100 (1997) & D-Sorbitol & 50 & 7.0 & $13.1 \%$ \\
\hline G.oxydans (pGUC-k0203-GS-k0095-pqqABCDE) (2014) & D-Sorbitol & 150 & 39.2 & $24.5 \%$ \\
\hline G. oxydans-ss-pqqABCDE (2016) & D-Sorbitol & 150 & 44.5 & $27.8 \%$ \\
\hline
\end{tabular}


2017; Jin et al., 2019), G. oxydans itself is not suitable for highthroughput screening of efficient SDH. E. coli could be a common host for the high-throughput screening of enzymes by using error-prone PCR.

By optimizing the promoters and the host of E. coli, it was found that not only the conversion ratio of L-sorbose to 2KLG was low, there was also some byproducts that may be associated with the SDH. In order to decrease the accumulation of by-product, several means have been attempted, such as coexpression of SNDH (Fu et al., 2007; Du et al., 2013), blocked the potential competition pathway of L-sorbose to 2-KLG by knocking out the aldosterone reductase genes (Yum et al., 1998; Penning, 2015). According to reports in the gene of Klebsiella, the transport of L-sorbose into bacteria requires the L-sorbosespecific phosphotransferase system (PTS) (Slater et al., 1981; Wehmeier et al., 1995; Yebra et al., 2000). Therefore, knockout of PTS related genes in E. coli has also been attempted, including PtsG (glucose phosphoryl transferase) (Han et al., 2004), FruA (fructose phosphoryl transferase), PfkA (phosphofructokinase) (Vinopal et al., 1975), PtsH (phosphorylated carrier protein), PtsI (phosphotransferase I) (Woodward and Charles, 1982), and GlcA (glycolate transporter) (Sprenger and Lengeler, 1984). Though the interactions of SDH with these genes remains unclear, our results demonstrated that knockout of some of the genes could significantly enhance the function of $\mathrm{SDH}$ in E. coli, which could benefit the high-throughput screening of enhanced SDH in E. coli.

After investigated a majority of the SDHs reported in $G$. oxydans in our lab, the enzyme activity in either G. oxydans or E. coli is far away from the requirement for competition with the current industrial scale vitamin $\mathrm{C}$ process. Though it has been reported that the $G$. oxydans strain can be directly mutagenized to enhance its performance in 2-KLG production (Park et al., 2012; Zhu et al., 2012; Yang et al., 2017), there was no reports on the directed evolution of SDH from G. oxydans. In this study, it was found that only overexpression of SDH in E. coli BL21 (DE3) could produce 2 -KLG. Though the current mixed fermentation system for the production of 2-KLG has been extensively studied
(Takagi et al., 2010; Yang et al., 2015), production of 2-KLG in E. coli are rarely investigated. The results presented here could provide a useful reference for the metabolic engineering of $\mathrm{L}$ sorbose to 2-KLG in E. coli. In this work, overexpression of a FAD-dependent SDH from G. oxydans WSH-004 in E. coli was studied. Owing to its unique characteristics, this SDH could be used to directly produce 2 -KLG from L-sorbose with high substrate/product specificity. A platform strain suitable for highthroughput screening of SDH was constructed. The screening platform strain constructed here should be a model strain suitable for further enhancing the production of 2-KLG by either $G$. oxydans or other bacteria.

\section{DATA AVAILABILITY STATEMENT}

The raw data supporting the conclusions of this article will be made available by the authors, without undue reservation, to any qualified researcher.

\section{AUTHOR CONTRIBUTIONS}

JZ and JC provided the main core ideas of the experimental design. WZ, LL, and XS mainly searched for references, designed of experimental methods, purchase of consumables and reagents used in the experimental process, operated experiment and analysis of data, drawing of charts, and writing of articles.

\section{FUNDING}

This work was supported by grants from the National Natural Science Foundation of China (Key Program, 31830068), the National Science Fund for Excellent Young Scholars (21822806), the Fundamental Research Funds for the Central Universities (JUSRP51701A), the National First-class Discipline Program of Light Industry Technology and Engineering (LITE2018-08), the Distinguished Professor Project of Jiangsu Province, and the 111 Project (111-2-06).

\section{REFERENCES}

Anderson, S., Marks, C. B., Lazarus, R., Miller, J., Stafford, K., Seymour, J., et al. (1985). Production of 2-Keto-L-Gulonate, an intermediate in L-Ascorbate synthesis, by a genetically modified Erwinia herbicola. Science 230, 144-149. doi: $10.1126 /$ science.230.4722.144

Carr, A. C., and Maggini, S. (2017). Vitamin C and immune function. Nutrients 9:25. doi: 10.3390/nu9111211

Chen, Y., Liu, L., Shan, X., Du, G., Zhou, J., and Chen, J. (2019). Highthroughput screening of a 2-Keto-L-Gulonic acid-producing Gluconobacter oxydans strain based on related dehydrogenases. Front. Bioeng. Biotechnol. 7:385. doi: 10.3389/fbioe.2019.00385

Du, J., Bai, W., Song, H., and Yuan, Y. J. (2013). Combinational expression of sorbose/sorbosone dehydrogenases and cofactor pyrroloquinoline quinone increases 2-keto-L-gulonic acid production in Ketogulonigenium vulgare-Bacillus cereus consortium. Metab. Eng. 19, 50-56. doi: 10.1016/j.ymben.2013.05.006

Fu, S., Zhang, W., Guo, A., and Wang, J. (2007). Identification of promoters of two dehydrogenase genes in Ketogulonicigenium vulgare DSM

4025 and their strength comparison in K-vulgare and Escherichia coli. Appl. Microbiol. Biotechnol. 75, 1127-1132. doi: 10.1007/s00253-0070930-z

Gao, L., Hu, Y., Liu, J., Du, G., Zhou, J., and Chen, J. (2014). Stepwise metabolic engineering of Gluconobacter oxydans WSH-003 for the direct production of 2-keto-L-gulonic acid from D-sorbitol. Metab. Eng. 24, 30-37. doi: 10.1016/j.ymben.2014.04.003

Gao, Y., Zeng, W., Zhou, J., and Chen, J. (2017). Purification and characterization of L-sorbose dehydrogenase and L-sorbosone dehydrogenase from Ketogulonicigenium vulgare WSH-001. Wei Sheng Wu Hsueh Pao Acta Microbiol. Sin. 57, 1546-1554. doi: 10.13343/j.cnki.wsxb.20160499

Han, C., Zhang, W. C., You, S., and Huang, L. Y. (2004). Knockout of the ptsG Gene in Escherichia coli and cultural characterization of the mutants. Chin. J. Biotechnol. 20, 16-20. doi: 10.13345/j.cjb.2004.01.004

Jiang, Y., Chen, B., Duan, C., Sun, B., Yang, J., and Yang, S. (2015). Multigene editing in the Escherichia coli genome via the CRISPR-Cas9 system. Appl. Environ. Microbiol. 81, 2506-2514. doi: 10.1128/AEM.04023-14

Jin, C., Hou, W., Yao, R., Zhou, P., Zhang, H., and Bao, J. (2019). Adaptive evolution of Gluconobacter oxydans accelerates the conversion rate of 
non-glucose sugars derived from lignocellulose biomass. Bioresour. Techn. 289:121623. doi: 10.1016/j.biortech.2019.121623

Kim, T. S., Hui, G., Li, J., Kalia, V. C., Muthusamy, K., Sohng, J. K., et al. (2019). Overcoming NADPH product inhibition improves Dsorbitol conversion to L-sorbose. Sci. Rep. 9:815. doi: 10.1038/s41598-01948082-8

Liu, L., Li, Y., Zhang, J., Zhou, Z., Liu, J., Li, X., et al. (2011a). Complete genome sequence of the industrial strain Ketogulonicigenium vulgare WSH-001. J. Bacteriol. 193, 6108-6109. doi: 10.1128/JB.06007-11

Liu, L., Li, Y., Zhang, J., Zou, W., Zhou, Z., Liu, J., et al. (2011b). Complete genome sequence of the industrial strain Bacillus megaterium WSH-002. J. Bacteriol. 193, 6389-6390. doi: 10.1128/JB.06066-11

May, J. M., and Qu, Z. C. (2005). Transport and intracellular accumulation of vitamin C in endothelial cells: relevance to collagen synthesis. Arch. Biochem. Biophys. 434, 178-186. doi: 10.1016/j.abb.2004.10.023

Park, C. S., Yang, H. J., Kim, D. H., Kang, D. O., Kim, M. S., and Choi, N. S. (2012). A screening method for beta-glucan hydrolase employing Trypan Bluecoupled beta-glucan agar plate and beta-glucan zymography. Biotechnol. Lett. 34, 1073-1077. doi: 10.1007/s10529-012-0873-z

Penning, T. M. (2015). The aldo-keto reductases (AKRs): overview. Chem. Biol. Interact. 234, 236-246. doi: 10.1016/j.cbi.2014.09.024

Richter, N., Neumann, M., Liese, A., Wohlgemuth, R., Eggert, T., and Hummel, W. (2009). Characterisation of a recombinant NADP-dependent glycerol dehydrogenase from Gluconobacter oxydans and its application in the production of L-Glyceraldehyde. Chembiochem 10, 1888-1896. doi: 10.1002/cbic. 200900193

Saito, Y., Ishii, Y., Hayashi, H., Imao, Y., Akashi, T., Yoshikawa, K., et al. (1997). Cloning of genes coding for L-sorbose and L-sorbosone dehydrogenases from Gluconobacter oxydans and microbial production of 2-Keto-L-Gulonate, a precursor of L-ascorbic acid, in a recombinant G-oxydans strain. Appl. Environ. Microbiol. 63, 454-460. doi: 10.1128/AEM.63.2.454-460.1997

Slater, A. C., Jones-Mortimer, M. C., and Kornberg, H. L. (1981). L-Sorbose phosphorylation in Escherichia coli K-12. Biochim. Biophys. Acta. 646, 365-367. doi: 10.1016/0005-2736(81)90346-1

Sprenger, G. A., and Lengeler, J. W. (1984). L-Sorbose metabolism in Klebsiella pneumoniae and Sor+ derivatives of Escherichia coli K-12 and chemotaxis toward sorbose. J. Bacteriol. 157, 39-45. doi: 10.1128/JB.157.1.3945.1984

Takagi, Y., Sugisawa, T., and Hoshino, T. (2010). Continuous 2-Keto-l-gulonic acid fermentation by mixed culture of Ketogulonicigenium vulgare DSM 4025 and Bacillus megaterium or Xanthomonas maltophilia. Appl. Microbiol. Biotechnol. 86, 469-480. doi: 10.1007/s00253-009-2312-1

Vinopal, R. T., Clifton, D., and Fraenkel, D. G. (1975). PfkA locus of Escherichia coli. J. Bacteriol. 122, 1162-1171. doi: 10.1128/JB.122.3.1162-1171.1975

Wang, P., Zeng, W., Xu, S., Du, G., Zhou, J., and Chen, J. (2018). Current challenges facing one-step production of l-ascorbic acid. Biotechnol. Adv. 36, 1882-1899. doi: 10.1016/j.biotechadv.2018.07.006

Wang, P. P., Xia, Y., Li, J. H., Kang, Z., Zhou, J. W., and Chen, J. (2016). Overexpression of pyrroloquinoline quinone biosynthetic genes affects L-sorbose production in Gluconobacter oxydans WSH-003. Biochem. Eng. J. 112, 70-77. doi: 10.1016/j.bej.2016.04.011
Wehmeier, U. F., Wöhrl, B. M., and Lengeler, J. W. (1995). Molecular analysis of the phosphoenolpyruvate-dependent L-sorbose: phosphotransferase system from Klebsiella pneumoniae and of its multidomain structure. Mol. Gen. Genet. 246, 610-618. doi: 10.1007/bf00298968

Wenzel, U., Nickel, A., Kuntz, S., and Daniel, H. (2004). Ascorbic acid suppresses drug-induced apoptosis in human colon cancer cells by scavenging mitochondrial superoxide anions. Carcinogenesis 25, 703-712. doi: 10.1093/carcin/bgh079

Woodward, M. J., and Charles, H. P. (1982). Genes for l-sorbose utilization in Escherichia coli. J. Gen. Microbiol. 128, 1969-1980. doi: 10.1099/00221287-128-9-1969

Yang, W., Han, L., Mandlaa, M., Zhang, H., Zhang, Z., and Xu, H. (2017). A plate method for rapid screening of Ketogulonicigenium vulgare mutants for enhanced 2-keto-L-gulonic acid production. Braz. J. Microbiol. 48, 397-402. doi: 10.1016/j.bjm.2017.02.002

Yang, Y., Gao, M., Yu, X. D., Zhang, Y. H., and Lyu, S. X. (2015). Optimization of medium composition for two-step fermentation of vitamin $\mathrm{C}$ based on artificial neural network-genetic algorithm techniques. Biotechnol. Biotechnol. Equip. 29, 1128-1134. doi: 10.1080/13102818.2015.1063970

Yao, R., Hou, W., and Bao, J. (2017). Complete oxidative conversion of lignocellulose derived non-glucose sugars to sugar acids by Gluconobacter oxydans. Bioresour. Technol. 244, 1188-1192. doi: 10.1016/j.biortech. 2017.08.078

Yebra, M. J., Veyrat, A., Santos, M. A., and Pérez-Martínez, G. (2000). Genetics of L-sorbose transport and metabolism in Lactobacillus casei. J. Bacteriol. 182, 155-163. doi: 10.1128/jb.182.1.155-163.2000

Yum, D. Y., Lee, B. Y., Hahm, D. H., and Pan, J. G. (1998). The yiaE gene, located at 80.1 minutes on the Escherichia coli chromosome, encodes a 2-ketoaldonate reductase. J. Bacteriol. 180, 5984-5988. doi: 10.1128/JB.180.22.5984-5988.1998

Zhou, J., Du, G., and Chen, J. (2012). Metabolic engineering of microorganisms for vitamin C production. Subcell. Biochem. 64, 241-259. doi: 10.1007/978-94-007-5055-5_12

Zhou, S., Ding, R., Chen, J., Du, G., Li, H., and Zhou, J. (2017). Obtaining a panel of cascade promoter-5'-UTR complexes in Escherichia coli. ACS Synth. Biol. 6, 1065-1075. doi: 10.1021/acssynbio.7b00006

Zhu, Y. B., Liu, J. D., Liu, J., Du, G. C., Zhou, J. W., and Chen, J. (2012). A high throughput method to screen companion bacterium for 2-keto-L-gulonic acid biosynthesis by co-culturing Ketogulonicigenium vulgare. Process Biochem. 47, 1428-1432. doi: 10.1016/j.procbio.2012.05.010

Conflict of Interest: The authors declare that the research was conducted in the absence of any commercial or financial relationships that could be construed as a potential conflict of interest.

Copyright $\odot 2020$ Shan, Liu, Zeng, Chen and Zhou. This is an open-access article distributed under the terms of the Creative Commons Attribution License (CC BY). The use, distribution or reproduction in other forums is permitted, provided the original author(s) and the copyright owner(s) are credited and that the original publication in this journal is cited, in accordance with accepted academic practice. No use, distribution or reproduction is permitted which does not comply with these terms. 